ankle in a layer of fine cotton-wool, applied a piece of moistened pasteboard to the sole of the foot, extending from a little beyond the heel to half an inch in front of the great toe, broad enough to embrace the tread of the foot. Across the ankle I placed two diagonal strips of moistened pasteboard, and fixed the whole with smoothly, but steadily, compressing bandage. The dressing was conipleted by fixing with a few turns of bandage a dry pasteboard splint to the sole of the foot, and securing the lower part of the limb in the hollow of a pillow, tied round it with a couple of pieces of bandare. The patient was immediately comfortable, and continued so. He passed a good night, and when I saw him next day was quite easy. On removing the dressing after twenty-four hours, the toe was comparatively pale and shrunken, and could be handled comfortably. I reapplied the same dressing, but with firmer pressure. Forty-eight hours later improvement continued without interruption. I found the toe still further reduced, and as the sore, though cleaner, was not yet quite clean, I brushed over it, and into the crevices at its root and sirles, with a fine camel-hair pencil dipped in the following lotion:-Borax, half a drachm; compound tincture of lavenler, a drachm and a half; glycerine an ounce and a half; water, six ounces. This application did not cause the least pain; and when $I$ had once more immobilised and compressed the foot, I was able to drop it from the height of a couple of feet on to the couch without the least discomfort.

Valuable as dry dressing is in all recent wounds, lotions, similar to the foregoing in composition, are of essential service, when rightly employed, in foul wounds and old ulcers. The term "detergent" of the old surgeons has lost none of its fitness, and the therapeutic agents which it designates have lost none of their practical usefulness.

In the case (5) now under consideration the result was largely due to absolute rest. The value of rest no one questions; but what is needed is a more clear and precise understanding of the means to be adopted to carrv it into effect. Rest, to be of real service in the multitude of sargical cases which are benefited by it, must be, as nearly as practicable, absolute; and the construction of immovable apparatus, suitable to the exigencies of particular cases, will be found to tax the ingenuity, and to demand the nicest combination of gentleness and strength. Pressure was another great factor in my patient's improvement. It was so partly, no doubt, as an aid to immobilisation, in unifying the parts of the apparatus and fixing the ankle and the smaller joints of the foot. But it must always be borne in mind that, whereas comparatively slight pressure, if hard and circumscribed, is painfal; uniform, smooth, elasti pesssure, accurately applied, is most soothing. It acts by controlling the local circulation, preventing extrarasation, promoting absorption of interstitial deposits, and lulling, to impotence for mischief, muscular spasm-that most potent cause of local and constitutional unrest.

A wrist-joint, liot and swollen after a heavy fall on the hand; an ankle after a slip and a twist, grown big and mottled and tender as a boil; a woman's pendulous, hot, ten ler breast, breeding an abscess; the fleshy, jumping stump of an amputated thigh, are one and all relieved, as if by magic, under steady, uniform, elastic pressure. That pressure of which the surgeons of the ure-Hunterian enoch had so clear a conception, of which old Baynton of Bristol wrote so quaintly, John Bell and John Scott so eloquently yet so tersely, is all but forgotten. Little better has been the fate of the teaching of those modern classies, the elder Larrey and Seutin. Few understand the principle of, fewer still practise, pressure as inferior to none of the cardinal therapentic surgical agencies.

Rest and position, not as physiological abstractions, but as therapeutic agencies, carried out with all the resources and precision of surgical mechanics, are invaluable therapeutic resources, but it is only when combined with pressure that they enable the surgeon to realise the full power of vital endowment, to repair lesions and to resist the decaying tendencies inherent in dead organic matter.

Conceding the antagonism of putrefactive changes and vital processes, and the value of agents which prevent or neutralise the products of decomposition, it is narrowing the basis of surgical science, to the exclusion of some of its greatest resources, to concentrate the attention on antiseptic indications. On broad scientific grounds, Mr. Savory's protest against the expression "antiseptic surgery" claims assent. In the vast majority of wounds coaptation, once effected and steadily maintained, is followed by union. In such cases as a compound fracture, an amputation at the hip-joint, or ligature of an arterial trunk, frequent change great principle of REST, which, for local and constitutional reasons, is of the very essence of success. To assume as a general proposition, for revolutionising surgical practice, that infection exists ready made in the atmospluere, which only requires to be filtered of its germs as a condition precedent to the healing action, is to beg the whole question at issue, in the face of overwhelming clinical evidence to the contrary. Birmingham.

\section{WAYS OF REMEMBERING.}

\section{BY J. MORTIMER-GRANVILLE, M.D.}

THE fact that there is a practical difference between knowing a thing and being able to remember it is sure to be brought home to the student in any branch of science, very early in his career. What precisely is the nature of this difference, and how is it to be adjusted? Before we try to find answers to these homely but earnest questions, let us expose and put out of the way a source of misconception which often occasions trouble and disappointment to minds admirably fitted for intellectual work, but inexperienced in the exercise of their powers and faculties. A man of acute and clear perception, endowed with a quick understanding, will comprehend a subject, take it in with a rapid mental glance, and seem to have made it his own. He "learns easily," but, alas, he forgets with even greater facility. The truth is that he has never learnt in any mnemonic sense. What he has done is to apprehend; and although the brain is undoubtedly capable of a process analogous to instantaneous photographing, it rarely performs this function at the behest of the will, unless it has been specially trained to do so ; or when it does thus instantly receive an impression, the record is not permanent. The faculty of instantaneous mental photography is more commonly the agent of the sub. consciousness than of the supreme consciousness, and it takes in the impressions we would gladly have effaced, while those it is desired to retain are obliterated almost as soon as they are registered. Apprehension is a function of the intellect which may be, and in the case of what are called "clever" persons often is, developed to a high deoree of efficiency without any corresponding exercise of the recording faculty. Just as a man may work out a problem or perform an aritlinetical calculation with perfect command of the data and processes involved, but in no way burden his mind with the details, or even the result of his work, if these do not personally concern him, he may concentrate attention and bring his reasoning faculties to bear on a subject of study and master its details, so as to obtain a clear comprehension of the whole while he is not reoistering any impression to form the basis of memory. Indeed, it is a notable circumstance that in a large class of minds the faculty of apprehension is developed, so to say, at the cost of that of mental registration or memory the whole force of the intellect being, as it were, expended in understanding, while the storing of impressions is left to chance, which generally means that it is wholly neglected. It is therefore important to bear in mind that a quick understanding does not either involve or imply an aptitude for study. It is simply an effective power of perception, and is not uncommonly associated with a proneness to forget, which is in truth the effect of an absence or inefficiency of the faculty of mental recording. The distinctness and almost antagonism of these two functions of the mind, anderstand ing and memory, is curiously apparent in the fact that idiots have often extraordinary powers of retention and recollec tion, while the most intelligent hearers and readers often find to their cost that they are the most forgetful. The student should not allow the consciousness he may have of a quick understanding to encourage him in the neglect to cultivate his memory, or be misled by a "good memory" to assume that he is endowed with high intellectual ability.

It is, undoubtedly, possible that the mind may be duly charged with a record of any subject or any information, and be unable to remember it at will. This circumstance arises from the fact that memory concerns the method of recording rather than the record itself. A piece of know. 
ledge-if I may use the term-is often put away safely in the archives of memory, but no care has been taken to mark the place or leave a clue for its recovery when wanted. It may turn up at any moment, but cannot be produced by the will, for the simple reason that will has not been concerned in putting it away, or is not orderly in its action and trained to the task of re-collection. The difference between knowing a thing and being able to remember it is the difference between having a piece of property and lnowing where to find it. The way to adjust this difference is to make the act of recording impressions a part of the process of receiving and shaping them. This is what some persons try to do by a recourse to what are known as "technical memories." Those who attach value to these devices must bear with me when I contend that they are not in harmony with the laws of psychology, and are therefore unscientific. It would be absurd to assert that they are useless, because everyone knows that "bodf" has helped many a bewildered student to remember that the descending horn of the cerebral ventricle has a course backwards, outwards, downwards, forwards, and inwards; but clumsy and needless tools have often been employed in good work and, nevertheless, wisely thrown aside when it was found that the work could be done much better without them. The natural and only true basis of memory is a well formed impression. It is not essential that the impression should be fully understood at the time it is made, or the subject-matter wholly mastered by the understanding, but if the record is to be found by thought at pleasure, it must be registered by thought, and in such a way as to be easily recoverable. If the will is to contro] the act of recollection it must be directly concerned in that of recording, and it will greatly facilitate the cultivation of a "serviceable memory" if the processes of apprehending and recording are studied together and intelligently combined or correlated.

The organ of the mind is connected by several lines of communication with the external; and impressions-or more accurately the agents of impression-travel more freely along some than others. One man will be more effectively impressed by what he hears than by what he sees, and so on. Probably, the deepest, clearest, and most permanent impressions are those made when the subject-matter is communicated to the brain by several senses at the same instant, or at least during the same observation. For example, the impression received from an object that can be seen, heard, felt, and perhaps brought under cognisance by the olfactory sense simultaneously, will be deeper and clearer than that of another object which can only be recognised by a single perceptive faculty. Nevertheless, most persons have a special aptitude for receiving impressions through some particular avenue of the senses, and it is importan for the student to ascertain which is the most open and sensitive of the lines of communication in his individual case. A simple experiment, which may be described as follows, will, if carefully performed, supply the information desired. Let some other person write on a slip of paper, which we will call No. 1, six or eight familiar words con sisting of a single syllable, in such order that they shall not have any connections of meaning or sense. ${ }^{1}$ Let him next write the same words in a different order on another pape (No. 2), again avoiding any arrangement which might connect the words in thought by sound, sense, or meaning. The person to be experimented upon must neither have seen nor heard the contents of the two papers, or know the precise number of the words written. Now place the paper, No. 1 , before him, and let him read it once only, and silently, so that no element of sound may be imported into the experiment. Then let the paper be removed, and let him at once write down from memory the words just read in their order. This should be done as quickly as possible, because the purpose of the experiment is not to test the permanence of the impression, but the readiness and clearness with which it is formed. As soon as he has written all he can recollect, remove the paper, without waiting for any correction or addition, and mark it No. I for reference hereafter. Next let the person who wrote the words on paper No. 2 read them once, clearly, at an ordinary rate, neither too fast nor tou slow, and with.

1 If the number of words suggested should prove too great in any case a smaller one may be employed, but it is necessary that the worls should be arranged so as to avoid any connexion, or the result will be misleading; the ideas will be less in number than the words, whereas it is essential that each word should represent an independent and readily combine to represent compound ideas, and for our immediat purpose we must use simple ones. out any particular emphasis, and let the student after he has concluded immediately write down what he can remember. The result must be quickly removed with the same precaution as before, to avoid after-thoughts, and the paper marked No. 2. The process is then to be repeated in an inverse order, the words chosen being different, and the arrangement as heterogeneuus as in the first stage of the experiment, but the oral reading being given first, and the paper which is read at sight subsequently. The papers containing the results are to be marked with the numbers corresponding to the test papers. The two sets of papers are now to be carefully compared, and a judgment found on the basis of general accuracy as to the words and their order. If no great difference is apparent in the results obtained, the experiment may be repeated with any variation, such as the substitution of figures for words, but the conditions must be carefully adjusted, so that memory is not helped, or the burden of the trial will be thrown on the recollection instead of on the faculty of receiving impressions. It will not generally be difficult to form a clear notion of difference between the aptitude displayed for re ceiving by ear and by eye respectively, and the readiest faculty will be the one on which it is safest to rely so far as taking in knowledge is concerned. The man who appre ciates most readily by ear should not, as a rule, take notes at lecture, whatever he may do afterwards; while the student who is not so expert in receiving notions from the ear as by the eye may do wisely to take down a lecture in shorthand, or even write a full note, by way of impressing it on his mind. The latter will also probably find that he derives more advantage, generally, from reading books than from oral instruction.

It is, however, necessary to know more than this experiment has, so far, communicated. The faculty of reception is one thing, that of retention another. In order to explore the latter, it will be desirable to repeat the experiment already described with new word materials, and to allow an interval of, say, half an hour to elapse between the reading by sight and writing from memory, in the one case, and the same time between the dictation and writing in the other. Great care must be taken to render the intervals as nearly as possible equally distractive as regards the way the mind is employed, and by reversing the order of the sight and sound tests, as previously indicated, to correct any error likely to creep in from the fact that when the same words are brought a second time under comnisance they are, of course, recent or familiar. This further experiment will throw new light on the comparative efficiency of the two faculties as recording agents, but to obtain the full information required, the four sets of test and result papers must now be examined from another standpoint. The nature of the mistakes made is not less suggestive than the relative amount of acca racy. As a rule, persons who habitually remember by earthat is, by calling to mind a mental record of sound-will, when writing from memory, make mistakes suggested by similarity of sounds; the words written, if not the right ones, will be of somewhat like sound; while specially softsounding words or syllables are likely to be omitted. On the other hand, those who remember by pictures of thought or mental characters are notably apt to substitute word. that bear a general resemblance to each other in their own caligraphy, and to drop words or parts of words, as though writing hastily from an ideal copy. A very little reflection will make it apparent that by bestowing a few hours to the scrutiny I have suggested any man may obtain a considerable and useful insight into his individual way of remembering, and on the hasis of the knowledge thus acquired he will be able to determine which of his powers it is best to cultivate. This initial point being settled, we may proceed to indicate briefly a few of the most practical nethods of developing the faculty it is proposed to train to the highest attainable excellence, which, of course, will vary with the inlividual energy, intellectual force, and perseverauce.

When the student knows that he takes in most accurately and remembers with the greatest readiness by sound, he should arrange his method of study so as to work this faculty directly. For example, he will be especially diligent in his attendance on lectures, and avoid as much as possible being distracted by sounds other than those which convey instruction. He will read aloud when studying in private, and impress the matters he desires to remember upon his mind by audible repetition, as a child learns his lesson by repeating it over to himself until he knows it. He will do more, bearing in mind the need of clues or threads of asso. 
'iaturn ly which to recover the matters put away in the main, he will take care to create for himself in the act of 1.araing a sullicient number of sound-linles which shall connert the fact: he desires to remember with others. The various teciuical formula constructed by teachers and recommended to the student fail first because they are not the creation of the mind which employs them; second, because they are nonnatural and arbitrary; and, third, because, the essential difference between a faculty of remembering by sound and liy sight not being recognised, the formulæ adopted are often uncongenial. Each student ought to make his own connecting links for ideas, and they should be natural, scientific, and appropriate to his special faculty. The man who remembers by sound will find it easier and better to remember a chemical decomposition, the characteristics of a boue, or the relations of an artery by some formula which connects them by sound than by trying to picture the subject, while the reverse will be the case with the man who remembers by sight. The latter must fancy he sees the object or recall to mind some written or printed description of it before he can remember the details. The man with a memory for sounds will do wisely to employ that faculty constantly. The voice of his teacher will help lim, the sounds of letters, even a sort of musical notation which he will construct for himself intuitively, will contribute to the efficiency of the service sound renders hin. Tho points for him to bear in mind are that in learning soundlinks or connexions must be formed, and in remembering the posture of mind should be one of mental listening, because it is through a thought-sound the matter will be brought back to the consciousness. When, on the other hand, sight is the readiest mode for the reception of impressions, and is also the medium of memory, the aim must be to picture every matter it is desired to remember either in the shape of an ideal image of the object, or a written or printed description of the subject.

Professors of memory, taking ad vantage of the large number of persons who remember by nental imagery, have developed the system of teaching by pictures to great perfection. With the aid of a few mental images of squares, or figures more or less simple, they will enable a very dull scholar to "recol. lect" the most complicated figures and dates, or a seemingly bewildering array of facts. The formula of memory is in all these cases associative, and the lines or spaces employed to fix the several objects of thought stand for the connecting links. Every way of remembering is, as I have said, a process of link-making, and when a simple formula can be made to serve a variety of purposes-acting as the frame for a multitude of pictures-the task is simplified, and the result, for a time at least, proportionately certain A picture-memory requires that the threads or connecting-links by which any subject is to be recovered shall be pictorial; and to make the process natural these links should be as far as possible the actual surroundings of the object to be remembered. The student who has a memory of this class should learn his anatomy from personal dissection, and it will help him greatly if he can draw so that the subjects of thought may be impressed on the mind by sketching. Such a man will do well to employ the pencil more than the pen, and even in the study of a subject like chemistry to employ diagrams largely. There is scarcely any topic which may not be illustrated by figuring of some kind, and the hieroglyphics employed ought to be of the student's own personal devising, except when a teacher has first presented the facts pictorially, in which case it is better to accept and adopt the original imagery, on account of the strength of first impressions, and the confusion that might be caused by changing the symbol. A common error into which beginners are apt to fall is to try to combine, and therefore confuse, the two methods of remembering--by sight and by sound. They should be kept carefully apart, and only one adoptedwhichever is found to be the most natural and susceptible of culture, in conformity with the law of development, which makes grow th and efficiency the fruits of exercise.

A close scrutiny of the results obtained by the experiments above suggested will show that there is a class of cases in which knowledge is received by one line of communication and remembered by another. The number of errors may be greater when writing from sight or sound respectively, while the nature of the mistakes made points to the other medium as the agency concerned in remembering. When this happens, it will be needful to cultivate the two faculties side by side, and this may seem to create a difficulty. In practice, however, it is easy to make the requisite discrimination, and after the learner has matured his method of study he will find that the doubling of the process really economises time by improving the quality of the work done. The feat to be accomplished is simple enough, and bears a close analogy to the procedure of an arithmetician, who "proves" his sum; havin r added, he subtracts, or the converse. So the learner by sight who remembers by sound must take in his subject by pictures or characters, and practise reproducing a verbal account of them. If he hears most readily and remembers by sight, he will do well to listen, and, as I have said, read aloud when studying, then come away, or close his book, and proceed to picture what he has been thinking about, and draw or write a description of the imagery, to impress it permanently. This is what hundreds of persons do unconsciously, and if the process be necessary in any case it will be found to be natural and easy.

There is nothing novel in these suggestions beyond the recommendation of a formal experiment to ascertain which of the several sense communications is the most available for practical purposes. All that I have indicated as desirable to do is done intuitively by expert scholars; but as intelligent and self-controlled beings, we ought to know the nature and purpose of every intellectual process, and to the young man entering on a career of special study it will be helpful to receive a few hints as to the best mode of procedure. It does not concern the student standing on the threshold of his field of inquiry to know in what the physical hases of memory consist-if, indeed, anyone is in a position to give him precise information on the subject,-but it is of use to him to be told how he remembers, and how to choose the readiest and most effective instrument for the task he has to accomplish. It is a higher and better thing to possess the power, and know where to acquire information when it is wanted, than to carry about knowledge as a pack horse bears his burden. At the same time there is so much which it is indispensable the student should learn to hold in memory, that I venture to offer these few hints as to "ways of remembering."

\section{THE ANTISEPTIC METHOD IN RELATION TO DRAINAGE OF THE PERITONEUM IN ABDOMINAL SURGERY.}

BY J. KNOWSLEY THORNTON, M.B., C.M., SURGEON TO THE SAMARITAN FREE HOSPITAL FOR WOMEN AND CHILDREN.

(Concluded from p. 480.)

CASE 7.- The patient was a-single woman, aged thirtyfive, with an enormous multilocular tumour. She came into hospital with well-marked symptoms of mischief in the cyst, which had been tapped some months previously, though only a small quantity of fluid was then obtained. She was jaundiced, and had a rapid, weak pulse; morning temperature generally over $99^{\circ}$, afternoon and evening from $101.4^{\circ}$ to $102^{\circ}$. The operation was a difficult one. The cysts (there were three or four much of a size) all contained a mixture of ovarian fluid and a brown clot-like material, with a distinctly unpleasant odour. I had to break up the septa, and pump this material out with my hand; much of it necessarily flowed over the wound, and though I was careful in washing it away and cleaning my hands in strong lotion as often as possible, I could not feel any confidence that I did not introduce causes of putrefaction into the peritoneum. Extensive and very vascular adhesions had to be separated, and both ligatures and cautery were used to control the hæmorrhage. The peritoneum also poured out serum freely, and I therefore decided to put in a tube in spite of a very careful cleansing of the abdominal cavity. The patient did not do well from the first; but at the second dressing there was no discharge in the sponge and very little in the tube. Not being satisfied with her general condition, and as to the asepticity of the operation, I left the tube in. Next dressing there was more discharge, then more, till it soaked through everything; the pulse getting weak and rapid, and flatus passing badly. At the eighth dressing, remembering the first case, and not sufficiently considering the difference in 